\title{
Muntingia calabura: a review of its traditional uses, chemical properties, and pharmacological observations
}

\begin{abstract}
Context: Different parts of Muntingia calabura L. (Elaeocarpaceae), or ñkerukup siamò in Malay, have been reported to possess medicinal value, supported by a number of scientific studies.

Materials and methods: Literature has been retrieved from a number of databases (e.g., Pubmed, Science Direct, Springer Link, etc.). General web searches were also carried out using Google and Yahoo search engines by applying some related search terms (e.g., Muntingia calabura, phytochemical, pharmacological, extract, and traditional uses). The articles related to agriculture, ecology, and synthetic work and those using languages other than English or Malay have been excluded. The bibliographies of papers relating to the review subject were also searched for further relevant references.

Results and discussion: The literature search conducted using the above-mentioned Internet search engines only lead to the identification of 36 journals published as early as 1987 . From the articles reviewed, M. calabura possessed various pharmacological activities (e.g., cytotoxic, antinociceptive, antiulcer, anti-inflammatory), which supported the folklore claims and could be attributed to its phytoconstituents.

Conclusion: Muntingia calabura possesses remarkable medicinal value, which warrants further and in-depth studies. Therefore, this review paper is presented to help guide researchers to plan their future studies related to this plant in the hope of isolating potential leads for future drug development.
\end{abstract}

Keyword: Elaeocarpaceae; Ethnomedicinal uses; Pharmacological activities; Phytoconstituents 